\title{
The role of artificial intelligence in paediatric cardiovascular magnetic resonance imaging
}

\author{
Andrew M. Taylor ${ }^{1,2}$
}

Received: 5 May 2021 / Revised: 13 August 2021 / Accepted: 5 October 2021 / Published online: 22 December 2021

(c) The Author(s) 2021

\begin{abstract}
Artificial intelligence (AI) offers the potential to change many aspects of paediatric cardiac imaging. At present, there are only a few clinically validated examples of AI applications in this field. This review focuses on the use of AI in paediatric cardiovascular MRI, using examples from paediatric cardiovascular MRI, adult cardiovascular MRI and other radiologic experience.
\end{abstract}

Keywords Artificial intelligence $\cdot$ Cardiovascular $\cdot$ Children $\cdot$ Heart $\cdot$ Magnetic resonance imaging

\section{Introduction}

As with all aspects of imaging, paediatric cardiac imaging is expected to undergo dramatic changes over the next decade in all aspects of the work, driven by the use of artificial intelligence (AI). A recent review of AI in the literature identified five health care areas where AI is expected to have a huge impact [1]: health care systems management, diagnostics, clinical decision-making, patient data and predictive medicine.

There are few examples of how AI is used in paediatric cardiac imaging. This review presents some of these advances and gives a flavour of the opportunities that $\mathrm{AI}$ and other technologies have to offer. I use the patient pathway for a paediatric cardiovascular MRI scan to highlight the areas that can be affected by AI - the processes of ordering, organising, performing and reporting a cardiovascular MR scan and how these data can then be utilised for research and patient benefit (Fig. 1). I also describe some of the ways that AI can impact echocardiography and cardiovascular CT.

Importantly, developments in AI should help move clinical practice from an era of evidence-based medicine to one

Andrew M. Taylor

a.taylor76@ucl.ac.uk

1 Great Ormond Street Hospital for Children, Zayed Centre for Research, 20 Guildford St., Room 3.7, London WC1N 1DZ, UK

2 Cardiovascular Imaging, UCL Institute of Cardiovascular Science, London, UK of intelligence-based medicine [2] and precision patient management, where information from big data is used to create knowledge that is then drilled down to meet the specific needs of the individual patient in real time. Ultimately, this is expected to improve patient outcomes as well as patient and clinician experiences. Ideally, it will also free clinician time from many of the mundane processes that we currently need to carry out.

\section{How artificial intelligence can affect the patient pathway in cardiovascular magnetic resonance imaging}

\section{Ordering, scheduling and protocolising}

The first step in any radiologic procedure is test ordering. The aim is to have the right test (most appropriate test for the clinical question) at the right time (clinical urgency, patient choice) that is efficient (minimising missed appointments and providing the most cost-effective test).

Improving the information on the imaging request form, which is often poor, is a crucial first step to drive all these processes. One recent study found that $80 \%$ of imaging requests did not have the relevant clinical information [3]. AI tools can extract clinical information from the clinical electronic patient record (EPR) to improve imaging modality selection [4] and then drive imaging protocolisation to address the relevant clinical questions [3]. One such machine learning tool was able to analyse unstructured text 


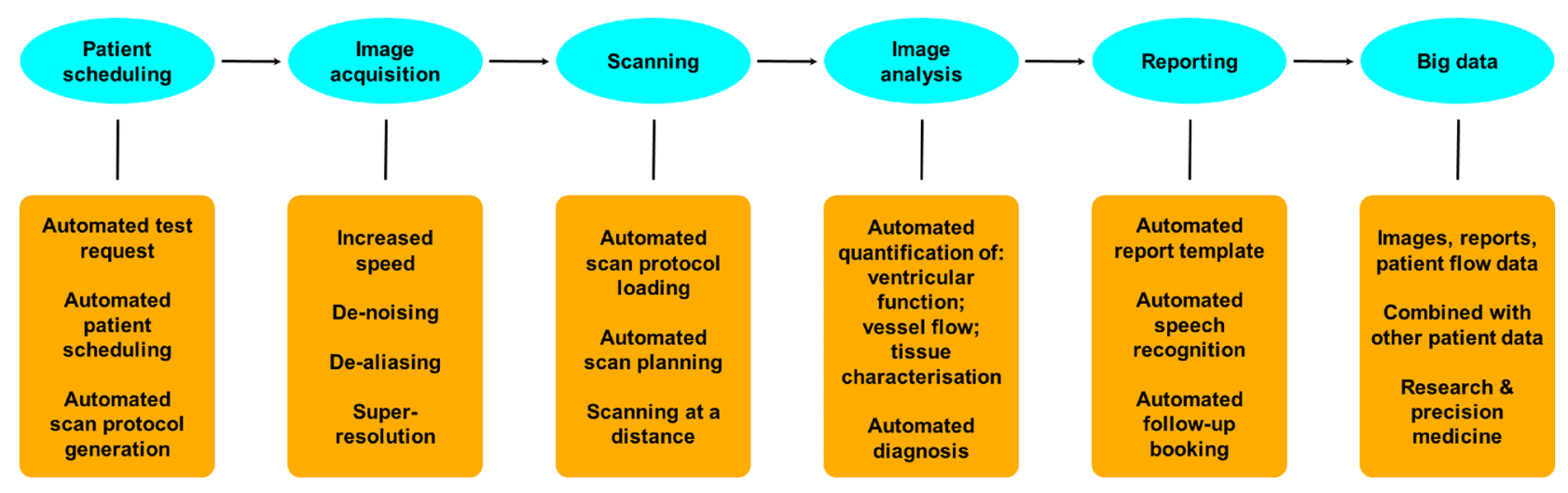

Fig. 1 Aspects of the radiologic patient pathway for paediatric cardiovascular MRI where artificial intelligence can play a role

for clinical indications for neurologic MR imaging requests and appropriately protocolise the MR scan with an accuracy of 95\% [5]. For cardiovascular MR imaging, prior clinical data and previous imaging protocols (from the individual or patients with similar underlying heart disease) could be used to potentially predict scan times. Furthermore, algorithms could be used to search the EPR to ensure that there are no contraindications to MRI, pre-populating the MR consent form with this information.

Algorithms can also be used to support optimisation of scheduling:

- to define optimal times for appointment bookings [6];

- to predict which patients might not attend [7]; and

- to define optimal follow-up times [8], automatically booking follow-up imaging.

This can improve efficiency - fewer staff calling, texting with appointment reminders, etc. - all of which can lead to an improved patient experience with more timely reminders about dates and times of imaging appointments, fewer missed appointments, improved utilisation of expensive scanner time and appropriate use of staff. Machine learning has also been used to predict wait times and delays on the day of imaging to keep patients and families better informed [9].

\section{Cardiovascular magnetic resonance sequences}

As we know, MRI is an inherently slow process. Hence, algorithms can reduce the amount of data required and support more rapid scanning. This is more so in cardiovascular imaging, where data are acquired not only in three dimensions, but also over time.

Multiple techniques have been developed over the years, including parallel imaging and compressed sensing, to reduce acquisition times for cardiovascular MR sequences.
These imaging techniques are prone to signal degradation and aliasing and require prior information, which is often crude at present. AI methods are excellent at discovering patterns and have been used to improve cardiovascular MR image quality for sparsely sampled data. Deep neural networks, in particular convolutional neural networks (CNNs) and recurrent neural networks, have been used to solve machine learning problems, predominately in a supervised way (using known ground-truth data) [10]. Examples include:

- de-noising data (to enable k-space under sampling),

- de-aliasing data (to enable k-space under sampling),

- super-resolution (to use low-resolution data to predict high-resolution detail), and

- reduced errors from field inhomogeneity and eddy currents (more accurate flow imaging).

In paediatric cardiovascular MR, clinical validated machine learning studies have shown that real-time acquisitions for two-dimensional (2-D) cine imaging can be used to reduce scan time from just under $5 \mathrm{~min}$ to $18 \mathrm{~s}$ with no loss in image quality [11]. This required training in 10 people with congenital heart disease (CHD). For three-dimensional (3-D) whole heart imaging in people with CHD, super-resolution machine learning reconstruction has been shown to reduce scan time from $8 \mathrm{~min}$ to $3 \mathrm{~min}$, with similar image quality compared to current methods [12]. Similar integration of AI for rapidly reconstructing four-dimensional (4-D) flow [13] and 3-D late-gadolinium enhancement [14], though not tested in the CHD setting, might also speed cardiovascular MR imaging.

Ultimately all these methods allow for image acquisition to be quicker, reducing the overall scan time by factors of up to 4 (60-min scan now performed in $15 \mathrm{~min}$ ). For paediatric cardiovascular MR, this allows for reduced need of general anaesthetic for younger children (shorter scans, no need for 
breath-holding; Fig. 2), better overall image quality from better compliance (again shorter scans, no need for breathholding) and increased scanner utilisation (more scans performed each session).

\section{Cardiovascular magnetic resonance scanning}

As described, AI might be able to help with cardiovascular MR protocolisation, which would enable protocols to be automatically loaded into the sequence list for the appropriate clinical indication (e.g., basic CHD protocol, heart failure protocol, cardiomyopathy protocol). Furthermore, learning-based algorithms can be applied to enable automated planning for the ventricular imaging planes for imaging the heart, which can take less than $10 \mathrm{~s}$ to complete [15]. Another potential way to reduce scan time might be to determine which sequences lead to pertinent clinical information in any given condition, again focusing the imaging protocol.

Similar automated algorithms using CNNs have been used for echocardiography and have included view identification based on validation of more than 8,000 manually segmented echocardiograms, with accurate imaging plane identification (96\% for parasternal long axis) [16].

These methods support imaging technicians as they plan cardiovascular MR scans, reducing operator dependence and enhancing the homogeneity of imaging protocols locally and across organisations to enhance data for multicentre studies.
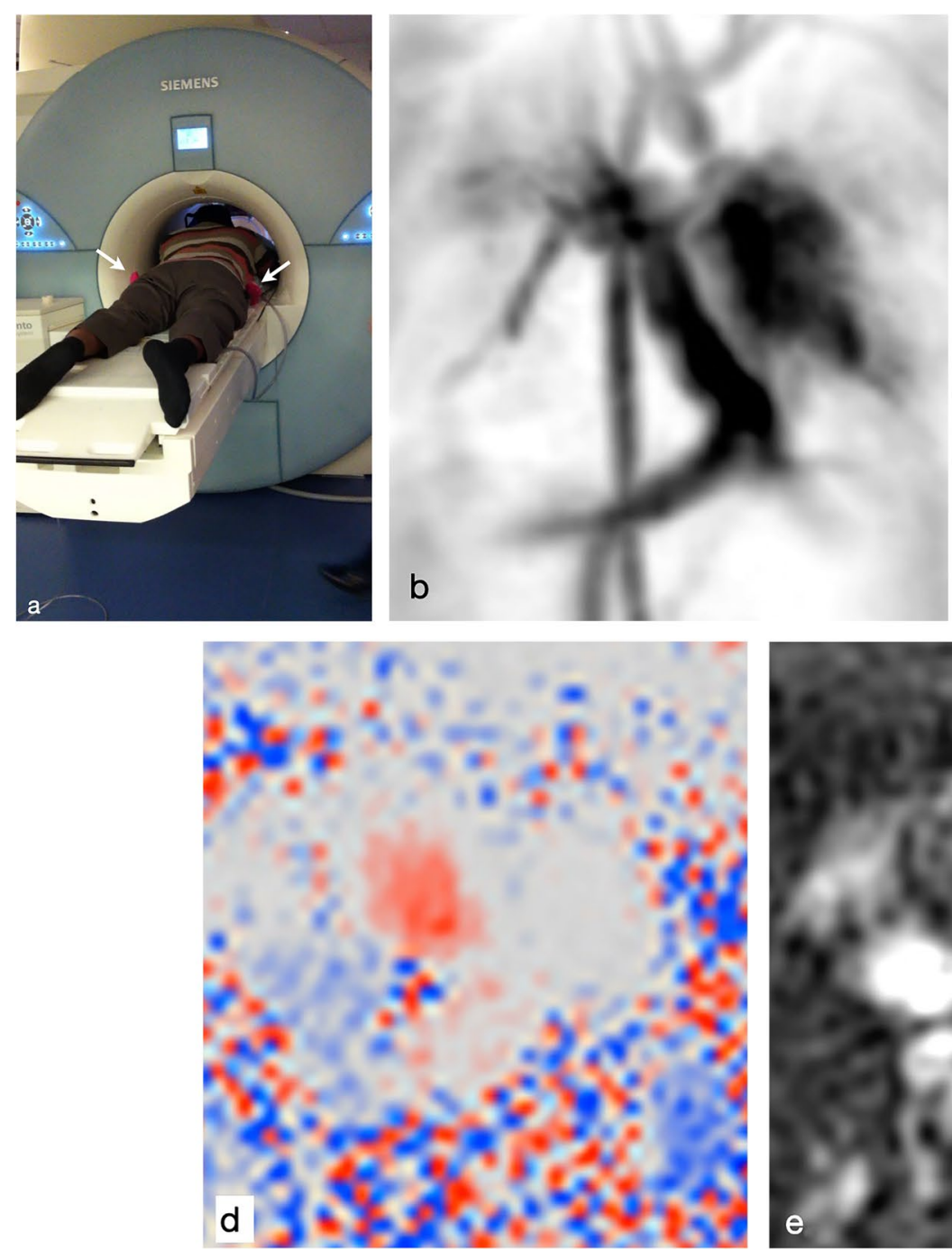

Fig. 2 Cardiovascular MR imaging in a 3-year-old girl with pulmonary hypertension without general anaesthetic. a Clinical image shows a father comforting his child (white arrows show small red socks of child!) during the scan, whilst child watches a video. b Anteroposterior time-resolved gadolinium-enhanced MR angiogram
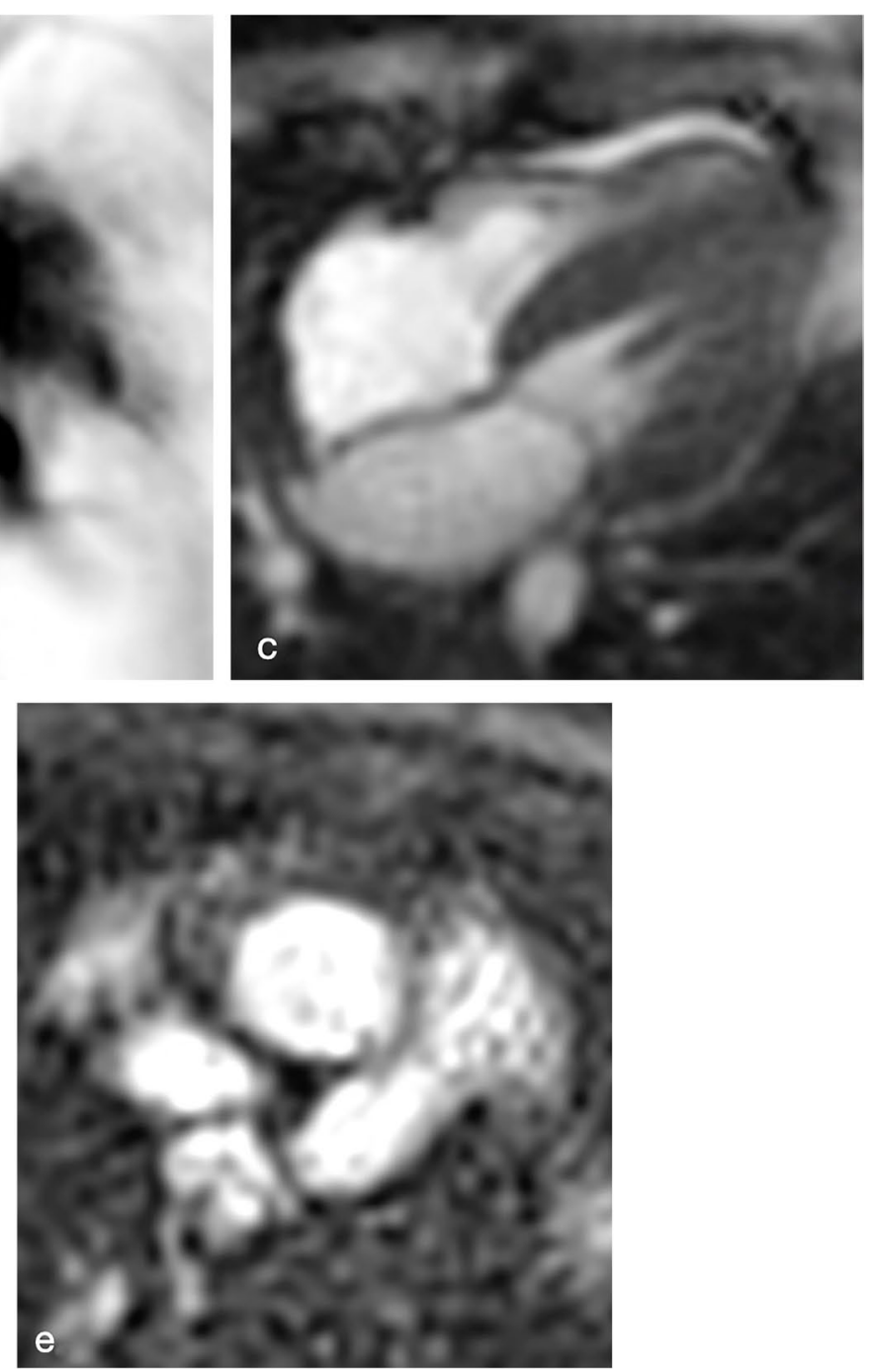

of the great vessels in the thorax. c Real-time cine imaging of the heart, 4-chamber view. d, e Oblique transverse phase-contrast velocity images (phase-contrast image [d], magnitude image [e]) of the ascending aorta to measure systemic flow 
This might mean that complex cardiovascular MR scans that require children to travel to distant specialist centres can be carried out to the same standard, utilising the same degree of expertise, using an MR scanner closer to the child's home - potentially reducing waiting times for scans and reducing travel times for patients and families.

\section{Cardiovascular magnetic resonance analysis}

\section{Imaging segmentation/data analysis}

As we continue to reduce the length of cardiovascular MR scans, benefiting patients, and increasing the number of scans we can perform, we remove one of the bottlenecks for cardiovascular MR waiting lists. However, the burden now shifts to the reporting environment. Currently, complex cardiovascular MR scans can take more than $60 \mathrm{~min}$ to report, with manual segmentation of ventricular volumes (dreaded circle drawing), flow post-processing (including 4-D flow), 3-D anatomy segmentation and tissue characterisation, all requiring significant cardiac imager time to analyse. It is therefore essential that these mundane tasks become more automated, to reduce reporting times and prevent a second bottleneck (no waiting for cardiovascular MR appointments because scans can be done quickly, but referrers now waiting for scan reports - the new bottleneck). Furthermore, algorithms that could compare a patient's studies to determine changes in volumes, flow, function and other imaging characteristics would be hugely useful, saving enormous amounts of time comparing studies and providing a trend for patient disease progression to track management (e.g., response to drug treatments, response to surgery or interventional cardiac catheterisation, or watchful waiting as part of routine surveillance).

Fortunately, a whole array of AI algorithms - machine and deep learning — are being developed to assist in data analysis, including automated quantification of ventricular volume, automated flow analysis, automated segmentation for tissue characterisation and automated cardiovascular MR tissues fingerprinting. They are described in the next paragraphs.

\section{Automated quantification of ventricular volume CNNs} using multi-vendor left ventricular (LV) data have shown excellent correlation $\left(r^{2} \geq 0.98\right)$ and agreement with measurement obtained by clinical experts [17]. Similarly good agreement has been found for LV volume and function assessment using a deep-learning-based algorithm for fully automated quantification [18]. Measurements of LV, left atrial (LA), right ventricular (RV) and right atrial (RA) volumes have also been shown to be accurate using a fully convoluted network with a large-scale annotated dataset, comparable to human interobserver variability using United
Kingdom biobank data [19]. Deep learning algorithms can now be integrated into frameworks for cardiovascular MR cardiac functional assessment, which can provide a degree of quality control. One such framework consists of preanalysis deep learning image quality control, followed by a deep learning algorithm for biventricular segmentation in long-axis and short-axis views, myocardial feature-tracking, and a post-analysis quality control to detect erroneous results [20]. The framework achieved good agreement with no clinical oversight, though some RV measures were less well performed by the framework than manual assessment. Importantly, deep learning ventricular segmentation of both the LV and RV has now been shown to be useable in CHD, though accuracy for RV measurements was worse than for LV measurements [21].

Automated flow analysis Although flow segmentation is relatively automated for the majority of cardiovascular MR post-processing tools, further automation to analyse images with limited or no operator input would be useful. A recent study looking at ascending aortic flow measurements at the aortic valve level has shown that accurate machine learning segmentation was uniformly successful, requiring no human intervention, with very high correlation $(r=0.99)$ between manual and algorithm segmentation and measurement in an external validation cohort [22].

Automated segmentation for tissue characterisation Lategadolinium enhancement can be used to guide clinical treatment, and clinical implementation of this information requires reproducible and reliable segmentation of the infarcted regions [23]. One automated method using deep CNNs to automatically quantify LV mass and scar volume in people with hypertrophic cardiomyopathy showed potential, with good agreement with manual segmentation [24].

Automated cardiovascular MR tissues fingerprinting Combining T1, T2, extracellular volume and late gadolinium enhancement data to create multi-parametric myocardial tissue characterisation has been demonstrated using pattern recognition algorithms to match the sampled signal to a predefined dictionary of predicted signal evolutions [25]. Such automated tissue characterisation could then be useful for automated diagnosis of cardiomyopathies and acute myocarditis and for the monitoring of cardiac transplant rejection.

\section{Automated diagnosis}

Automated diagnosis in CHD is not straightforward. One attempt has been to use an atlas-based computer-aided approach, which exploits similarity measures between the atlas and target images for normal cardiac anatomy and people with transposition of the great arteries following either 
the atrial or arterial switch operation. The method was validated using annotated images and subsequently showed a diagnostic accuracy of $97.3 \%$ when evaluated on a set of 60 whole heart MR images [26]. Deep learning algorithms have been shown to make the diagnosis of cardiac amyloid from cine cardiovascular MR and late-gadolinium images [27]. Such algorithms might then be able to differentiate between cardiomyopathies not only in adults, but also in children.

Considerable work is required to build and validate algorithms that can automatically diagnose CHD from 3-D images. However, as a first step, algorithms that can distinguish normal from abnormal cardiovascular MR images might be useful to ensure that complex CHD anomalies, which are rare and hence not seen often in routine clinical practice, are not missed, even if the exact diagnosis is not made, and that referral for expert opinion is carried out.

\section{Cardiovascular magnetic resonance report generation}

Algorithms could be used to generate a report template based on the clinical referral and the clinical patient information from the EPR. This information could also be used to search local and international clinical guidelines and research publications so that concurrent literature related to the case is instantaneously available at the time of reporting. For example, when reporting a case of Turner syndrome, the real-time availability of the current aortic dimensions from the latest consensus paper to guide clinical decisions would be hugely valuable.

Report generation can clearly be supported by automated speech recognition that either gathers data in a structured form or extracts data using natural language processing tools [28] to ensure that data are available for big data sets. The data from the radiologic report could also be used to automatically inform the referring clinician and used to drive follow-up recommendations - e.g., "Follow-up assessment for right ventricular volume assessment recommended in 2 years" automatically generates a cardiovascular MR appointment for the patient at this time point [29]. Furthermore, AI could be leveraged to generate targeted reports, specific for the referring cardiologist, the patient and the family, or the non-cardiologist, to ensure that the most pertinent information gets to the right person in the right language for them to understand and affect patient care.

\section{Provision of data for research and precision medicine}

The integration of data from multiple sources - clinical, imaging (radiology, digital pathology, photographs), genomic, proteomics and laboratory medicine - can be used to create vast databases that can be explored by AI algorithms (Fig. 3). After these databases have been explored

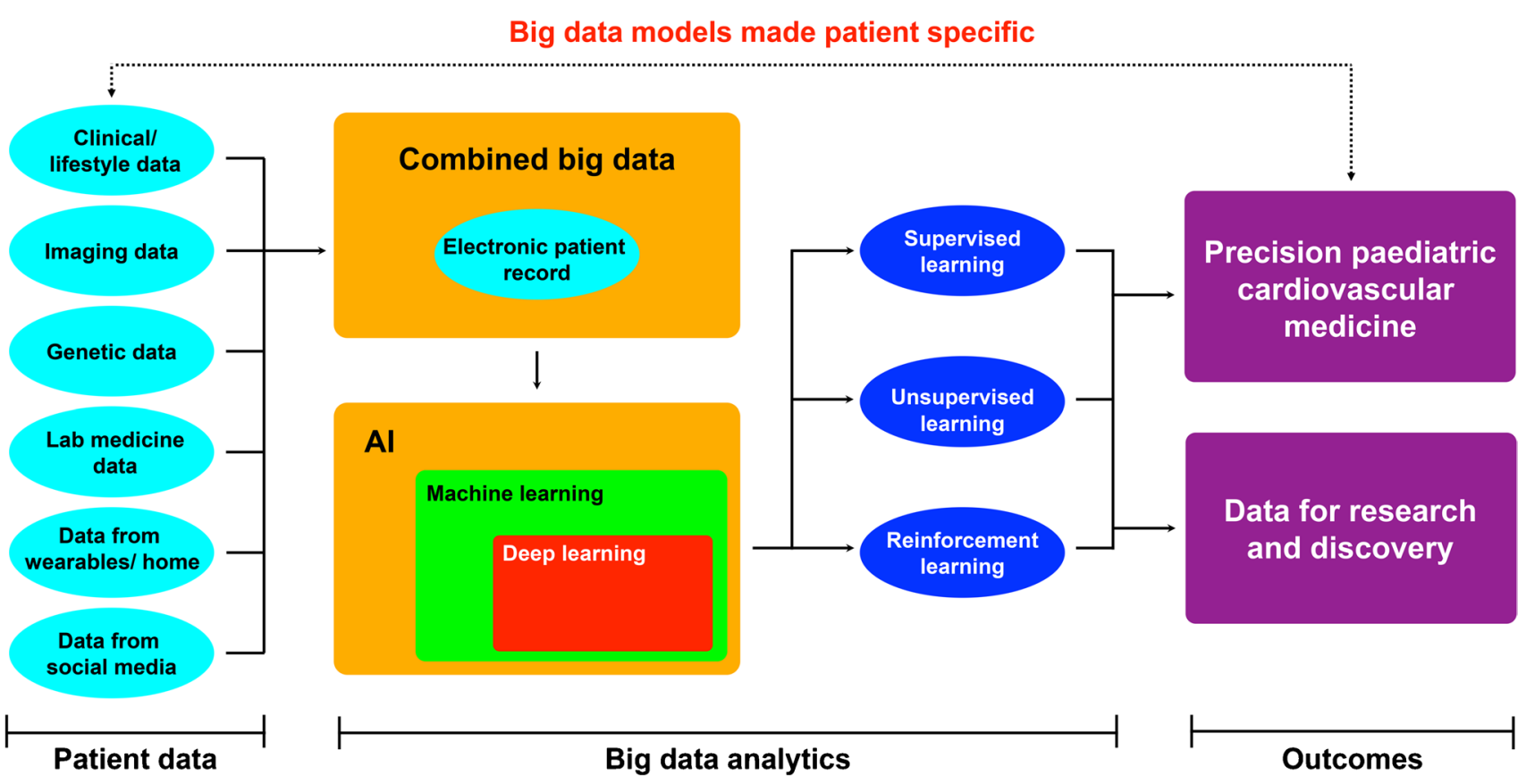

Fig. 3 Integration of patient information from multiple sources brought together in a single electronic patient record. These data, and data from thousands of other patients, are accessed using artificial intelligence (AI) and learning algorithms to create new understanding for patient populations. These big data can then be accessed for the individual patient, using AI and supervised, unsupervised and reinforcement learning to provide precision care in a feedback cycle, creating a virtuous cycle of patient data and care 
and validated, information can then be drilled down back to individual patients to drive precision medicine for each person based on their clinical history, imaging, laboratory findings and genetics, combined with the latest outcomes gathered in real time.

A few studies have used machine learning to combine cardiac imaging with other investigative and clinical features to predict prognosis. In one study, cardiovascular MR imaging data were combined with clinical, haemodynamic and functional markers in adults with pulmonary hypertension [30]. A machine learning survival model that included the cardiac motion parameters derived from cardiovascular MR was shown to have incremental prognostic power when compared with conventional parameters [30].

In a second study, measures of cardiac dimensions and function were derived from cardiovascular MR images of people with tetralogy of Fallot using automated deep learning analysis, which was combined with established clinical parameters and electrocardiogram (ECG) markers [31]. The deep learning models were trained on raw Digital Imaging and Communications in Medicine (DICOM) data at a single centre, then applied to a national cohort dataset to predict prognosis for adults with CHD.

\section{Summary of artificial intelligence in other areas of cardiovascular imaging}

Authors Xu et al. [32] gave an excellent summary of the AI developments in echocardiography and cardiovascular CT and the tools that have been developed.

\section{Echocardiography}

Artificial intelligence lends itself to all areas of echocardiography with automated analysis of chambers, ejection fraction and strain [16], valvular assessment [33] and aortic dimensions [34], with a move toward automatic diagnosis that has been shown in several areas, including the diagnosis of constrictive versus restrictive cardiomyopathy [35] and hypertrophic cardiomyopathy versus the athlete's heart [36].

\section{Cardiovascular computed tomography}

At present, the majority of algorithms that have been developed for cardiovascular CT have focused on the assessment of ischaemic heart disease with algorithms to support anatomical diagnosis, coronary artery plaque quantification, coronary blood flow and myocardial perfusion.

A combination of clinical and cardiovascular CT parameters using a machine learning framework has been shown to better predict 5-year mortality than conventional measures using the Framingham risk score [37]. Coronary artery blood flow - fractional flow reserve - can be calculated using computational fluid dynamics modelling [38] with excellent accuracy that has been demonstrated in several prospective multicentre studies.

Such algorithms might prove useful in the analysis of small stenosed vessels in CHD cardiovascular CT, and methods for automated CHD diagnosis developed for 3-D cardiovascular MR images should be useable in cardiovascular CT when they become available.

\section{Conclusion}

There are few concrete examples of the use of clinically validated AI in paediatric cardiac imaging. However, there is clearly huge potential to improve the experiences of children and clinicians in health care, using AI to drive precision patient medicine and disease discovery by combining siloed data sets from the whole patient pathway. Ultimately, this is expected to build an era of intelligence-based medicine that should enable more proactive, as opposed to reactive, care over time to support preventative medicine and early disease detection.

Much work remains to validate AI technologies in paediatric practice and to address some of these issues:

- changing the way care is delivered/clinical teams are managed;

- building good-quality (excellent data in) large data sets, which can be difficult in rare diseases;

- moving from single-centre validation to universal applicability;

- understanding data issues of confidentiality (patients and families in the paediatric setting), ownership (who owns health care data?), and value (what is health care data worth?);

- importance of regulation for ethical use of data, but not over-regulation that stifles crucial innovation;

- importance of understanding the role of decision support tools/models of care, and where AI information does not make sense; and

- the need for connectivity to link disparate data sets coming from the home, primary care and the hospital settings, and linking clinical teams, patients and families, and data scientists and engineers together.

If we can reduce the amount of mundane work we have to do, speed data acquisition and automate data analysis and diagnosis, whilst having all the relevant information at our fingertips to plan patient care based on the most recent guidelines, providing information that can then be used to drive the next person's 
care in a virtuous cycle, AI will ideally give us more time to think and talk to our patients and their families, paradoxically increasing the human touch that is so vital for patient care.

Acknowledgements A.M.T. is supported by University College London, Great Ormond Street Hospital for Children (GOSH) and the National Institute for Health Research GOSH Biomedical Research Centre. He receives funding from the British Heart Foundation.

\section{Declarations}

\section{Conflicts of interest None}

Open Access This article is licensed under a Creative Commons Attribution 4.0 International License, which permits use, sharing, adaptation, distribution and reproduction in any medium or format, as long as you give appropriate credit to the original author(s) and the source, provide a link to the Creative Commons licence, and indicate if changes were made. The images or other third party material in this article are included in the article's Creative Commons licence, unless indicated otherwise in a credit line to the material. If material is not included in the article's Creative Commons licence and your intended use is not permitted by statutory regulation or exceeds the permitted use, you will need to obtain permission directly from the copyright holder. To view a copy of this licence, visit http://creativecommons.org/licenses/by/4.0/.

\section{References}

1. Secinaro S, Calandra D, Secinaro A et al (2021) The role of artificial intelligence in healthcare: a structured literature review. BMC Med Inform Decis Mak 21:125

2. Chang A (2020) Intelligence-based medicine: artificial intelligence and human cognition in clinical medicine and healthcare. Academic Press, London

3. Lacson R, Laroya R, Wang A et al (2018) Integrity of clinical information in computerized order requisitions for diagnostic imaging. J Am Med Inform Assoc 25:1651-1656

4. Rousseau JF, Ip IK, Raja AS et al (2019) Can automated retrieval of data from emergency department physician notes enhance the imaging order entry process? Appl Clin Inform 10:189-198

5. Brown AD, Marotta TR (2018) Using machine learning for sequence-level automated MRI protocol selection in neuroradiology. J Am Med Inform Assoc 25:568-571

6. Richardson ML, Garwood ER, Lee Y et al (2020) Noninterpretive uses of artificial intelligence in radiology. Acad Radiol 28:1225-1235

7. Harvey HB, Liu C, Ai J et al (2017) Predicting no-shows in radiology using regression modeling of data available in the electronic medical record. J Am Coll Radiol 14:1303-1309

8. Lacson R, Wang A, Cochon L et al (2020) Factors associated with optimal follow-up in women with BI-RADS 3 breast findings. J Am Coll Radiol 17:469-474

9. Curtis C, Liu C, Bollerman TJ, Pianykh OS (2018) Machine learning for predicting patient wait times and appointment delays. J Am Coll Radiol 15:1310-1316

10. Montalt-Tordera J, Muthurangu V, Hauptmann A, Steeden JA (2021) Machine learning in magnetic resonance imaging: image reconstruction. Phys Med 83:79-87

11. Hauptmann A, Arridge S, Lucka F et al (2019) Real-time cardiovascular MR with spatio-temporal artifact suppression using deep learning - proof of concept in congenital heart disease. Magn Reson Med 81:1143-1156
12. Steeden JA, Quail M, Gotschy A et al (2020) Rapid whole-heart CMR with single volume super-resolution. J Cardiovasc Magn Reson 22:56

13. Vishnevskiy V, Walheim J, Kozerke S (2020) Deep variational network for rapid 4D flow MRI reconstruction. Nat Mach 2:228-235

14. El-Rewaidy H, Neisius U, Mancio J et al (2020) Deep complex convolutional network for fast reconstruction of 3D late gadolinium enhancement cardiac MRI. NMR Biomed 33:e4312

15. Lu X, Jolly MP, Georgescu B et al (2011) Automatic view planning for cardiac MRI acquisition. Med Image Comput Assist Interv 14:479-486

16. Zhang J, Gajjala S, Agrawal P et al (2018) Fully automated echocardiogram interpretation in clinical practice. Circulation 138:1623-1635

17. Tao Q, Yan W, Wang Y et al (2019) Deep learning-based method for fully automatic quantification of left ventricle function from cine MR images: a multivendor, multi-center study. Radiology 290:81-88

18. Bai W, Sinclair M, Tarroni G et al (2018) Automated cardiovascular magnetic resonance image analysis with fully convolutional networks. J Cardiovasc Magn Reson 20:65

19. Benjamin Böttcher B, Beller E et al (2020) Fully automated quantification of left ventricular volumes and function in cardiac MRI: clinical evaluation of a deep learning-based algorithm. Int J Cardiovasc Imaging 36:2239-2247

20. Ruijsink B, Puyol-Antón E, Oksuz I et al (2020) Fully automated, quality-controlled cardiac analysis from CMR: validation and large-scale application to characterize cardiac function. JACC Cardiovasc Imaging 13:684-695

21. Karimi-Bidhendi S, Arafati A, Cheng AL et al (2020) Fullyautomated deep-learning segmentation of pediatric cardiovascular magnetic resonance of patients with complex congenital heart diseases. J Cardiovasc Magn Reson 22:80

22. Bratt A, Kim J, Pollie M et al (2019) Machine learning derived segmentation of phase velocity encoded cardiovascular magnetic resonance for fully automated aortic flow quantification. J Cardiovasc Magn Reson 21:1

23. Karim R, Bhagirath P, Claus P et al (2016) Evaluation of stateof-the-art segmentation algorithms for left ventricle infarct from late gadolinium enhancement MR images. Med Image Anal 30:95-107

24. Fahmy AS, Rausch J, Neisius U et al (2018) Automated cardiac MR scar quantification in hypertrophic cardiomyopathy using deep convolutional neural networks. JACC Cardiovasc Imaging 11:1917-1918

25. Cavallo AU, Liu Y, Patterson A et al (2019) CMR fingerprinting for myocardial T1, T2, and ECV quantification in patients with nonischemic cardiomyopathy. JACC Cardiovasc Imaging 12:1584-1585

26. Zuluaga MA, Burgos N, Mendelson AF et al (2015) Voxelwise atlas rating for computer assisted diagnosis: application to congenital heart diseases of the great arteries. Med Image Anal 26:185-194

27. Martini N, Aimo A, Barison A et al (2020) Deep learning to diagnose cardiac amyloidosis from cardiovascular magnetic resonance. J Cardiovasc Magn Reson 22:84

28. Nath C, Albaghdadi MS, Jonnalagadda SR (2016) A natural language processing tool for large-scale data extraction from echocardiography reports. PLoS One 11:e0153749

29. Carrodeguas E, Lacson R, Swanson W, Khorasani R (2019) Use of machine learning to identify follow-up recommendations in radiology reports. J Am Coll Radiol 16:336-343

30. Dawes TJW, de Marvao A, Shi W et al (2017) Machine learning of three-dimensional right ventricular motion enables outcome prediction in pulmonary hypertension: a cardiac MR imaging study. Radiology 283:381-390 
31. Diller GP, Orwat S, Vahle J et al (2020) Prediction of prognosis in patients with tetralogy of Fallot based on deep learning imaging analysis. Heart 106:1007-1014

32. Xu B, Kocyigit D, Grimm R et al (2020) Applications of artificial intelligence in multimodality cardiovascular imaging: a state-of-the-art review. Prog Cardiovasc Dis 63:367-376

33. Aquila I, Gonzalez A, Fernandez-Golfin C et al (2016) Reproducibility of a novel echocardiographic 3D automated software for the assessment of mitral valve anatomy. Cardiovasc Ultrasound 14:17

34. Prihadi EA, vanRosendael PJ, Vollema EM et al (2018) Feasibility, accuracy, and reproducibility of aortic annular and root sizing for transcatheter aortic valve replacement using novel automated three-dimensional echocardiographic software: comparison with multi-detector row computed tomography. J Am Soc Echocardiogr 31:505-514

35. Mahmoud A, Bansal M, Sengupta PP (2017) New cardiac imaging algorithms to diagnose constrictive pericarditis versus restrictive cardiomyopathy. Curr Cardiol Rep 19:43
36. Narula S, Shameer K, Salem Omar AM et al (2016) Machinelearning algorithms to automate morphological and functional assessments in 2D echocardiography. J Am Coll Cardiol 68:2287-2295

37. Motwani M, Dey D, Berman DS et al (2017) Machine learning for prediction of all-cause mortality in patients with suspected coronary artery disease: a 5-year multicentre prospective registry analysis. Eur Heart J 38:500-507

38. Taylor CA, Fonte TA, Min JK (2013) Computational fluid dynamics applied to cardiac computed tomography for noninvasive quantification of fractional flow reserve: scientific basis. J Am Coll Cardiol 61:2233-2241

Publisher's note Springer Nature remains neutral with regard to jurisdictional claims in published maps and institutional affiliations. 